\title{
Gait and Axial Spondyloarthritis: Comparative Gait Analysis Study Using Foot-Worn Inertial Sensors
}

Julie Soulard ${ }^{1,2}$, PT, PhD; Jacques Vaillant ${ }^{1}$, PT, PhD; Athan Baillet ${ }^{3}$, MD, Prof Dr, PhD; Philippe Gaudin ${ }^{3}$, MD, Prof Dr, PhD; Nicolas Vuillerme ${ }^{1,4,5}, \mathrm{PhD}$

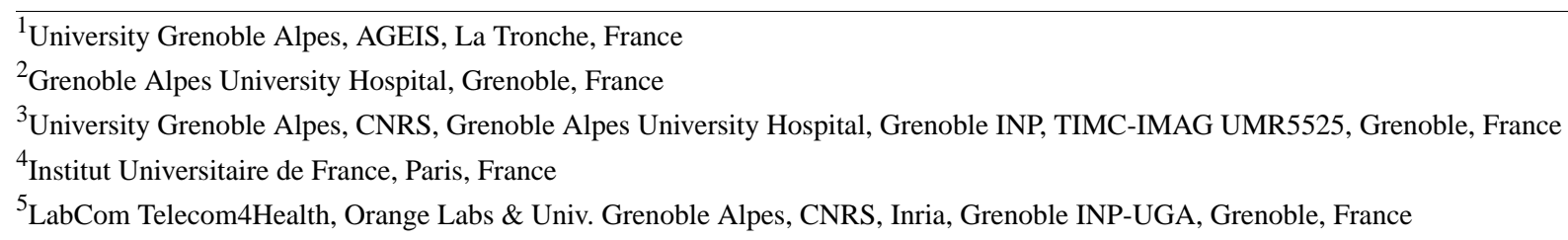

Corresponding Author:

Julie Soulard, PT, PhD

University Grenoble Alpes

AGEIS

Faculty of Medicine, Jean Roget Building

Place du commandant Nal

La Tronche, 38700

France

Phone: 33476637104

Email: juliesoulard.physio@gmail.com

\section{Abstract}

Background: Axial spondyloarthritis (axSpA) can lead to spinal mobility restrictions associated with restricted lower limb ranges of motion, thoracic kyphosis, spinopelvic ankylosis, or decrease in muscle strength. It is well known that these factors can have consequences on spatiotemporal gait parameters during walking. However, no study has assessed spatiotemporal gait parameters in patients with axSpA. Divergent results have been obtained in the studies assessing spatiotemporal gait parameters in ankylosing spondylitis, a subgroup of axSpA, which could be partly explained by self-reported pain intensity scores at time of assessment. Inertial measurement units (IMUs) are increasingly popular and may facilitate gait assessment in clinical practice.

Objective: This study compared spatiotemporal gait parameters assessed with foot-worn IMUs in patients with axSpA and matched healthy individuals without and with pain intensity score as a covariate.

Methods: A total of 30 patients with axSpA and 30 age- and sex-matched healthy controls performed a 10-m walk test at comfortable speed. Various spatiotemporal gait parameters were computed from foot-worn inertial sensors including gait speed in $\mathrm{ms}^{-1}$ (mean walking velocity), cadence in steps/minute (number of steps in a minute), stride length in $\mathrm{m}$ (distance between 2 consecutive footprints of the same foot on the ground), swing time in percentage (portion of the cycle during which the foot is in the air), stance time in percentage (portion of the cycle during which part of the foot touches the ground), and double support time in percentage (portion of the cycle where both feet touch the ground).

Results: Age, height, and weight were not significantly different between groups. Self-reported pain intensity was significantly higher in patients with axSpA than healthy controls $(P<.001)$. Independent sample $t$ tests indicated that patients with axSpA presented lower gait speed $(P<.001)$ and cadence $(P=.004)$, shorter stride length $(P<.001)$ and swing time $(P<.001)$, and longer double support time $(P<.001)$ and stance time $(P<.001)$ than healthy controls. When using pain intensity as a covariate, spatiotemporal gait parameters were still significant with patients with axSpA exhibiting lower gait speed $(P<.001)$, shorter stride length $(P=.001)$ and swing time $(P<.001)$, and longer double support time $(P<.001)$ and stance time $(P<.001)$ than matched healthy controls. Interestingly, there were no longer statistically significant between-group differences observed for the cadence $(P=.17)$.

Conclusions: Gait was significantly altered in patients with axSpA with reduced speed, cadence, stride length, and swing time and increased double support and stance time. Taken together, these changes in spatiotemporal gait parameters could be interpreted as the adoption of a so-called cautious gait pattern in patients with axSpA. Among factors that may influence gait in patients with 
axSpA, patient self-reported pain intensity could play a role. Finally, IMUs allowed computation of spatiotemporal gait parameters and are usable to assess gait in patients with axSpA in clinical routine.

Trial Registration: ClinicalTrials.gov NCT03761212; https://clinicaltrials.gov/ct2/show/NCT03761212

International Registered Report Identifier (IRRID): RR2-10.1007/s00296-019-04396-4

(JMIR Mhealth Uhealth 2021;9(11):e27087) doi: 10.2196/27087

\section{KEYWORDS}

ankylosing spondylitis; spondyloarthritis; gait; locomotion; pain; mobility; spatiotemporal; digital health; sensors

\section{Introduction}

The generic term spondyloarthritis $(\mathrm{SpA})$ regroups part of chronic inflammatory diseases with common clinical, genetic, and pathophysiological features [1,2]. Diagnosis of SpA is based on the Assessment of Spondyloarthritis International Society (ASAS) criteria [3]. Two groups of SpA are defined: axial SpA (axSpA) with main manifestations being on spinal and sacroiliac joints, and peripheral $\mathrm{SpA}$ with main manifestations being arthritis, enthesitis or dactylitis [1,3]. In this study, we will focus on axSpA which is divided into its radiographic (ankylosing spondylitis, AS) and its nonradiographic (nr-axSpA) forms $[1,2]$. Note that patients with axSpA could represent $0.13 \%$ to $1.4 \%$ of the world population [4].

Clinical manifestation of axSpA includes chronic inflammatory back pain and morning stiffness [5]. As a consequence of inflammation, structural damage can occur and lead to spinal mobility restrictions [5] associated with restricted lower limb ranges of motion [6], thoracic kyphosis [7], spinopelvic ankylosis [7], decrease in muscle strength [8], and sarcopenia [8]. It is well known that factors such as limited range of motion [9], reduced muscle strength [10], sarcopenia [11], thoracic kyphosis, and spinopelvic alignment [12] can have consequences on spatiotemporal gait parameters during walking.

It is interesting to note that a recent review concluded that no published work has investigated spatiotemporal gait parameters in patients with axSpA [13]. This is not the case for patients with AS, a subgroup of axSpA [3]. A recent review [14] reported that 21 articles assessed gait in AS. Interestingly, only 4 of them (19\%) used a healthy control group for comparison of spatiotemporal gait parameters [15-18]. What is more, results of these 4 studies are rather mixed and have reached somewhat inconsistent results and raised unanswered questions [15-18]. Some studies, indeed, reported gait impairment in patients with AS who presented with lower gait speed [18] and lower stride length $[16,18]$ than healthy controls. Other studies reported gait speed [15,17], stride length [15,17], cadence [16,17], swing time, and stance time percentages [18] of patients with AS similar to those of healthy controls. How can we explain these observed differences? It is possible that the relatively small sample size of these studies (from $n=10$ [17] to $n=18$ [18] in each group) represented an obstacle to the identification of any significant group differences. Note that this limitation is that of the authors themselves ("However, further study should be performed on a larger sample subjects" [15] and "the sample size was limited" [18]). It is also possible that self-reported pain intensity at the time of assessment played a role in these divergent results. On the one hand, it is recognized that low back pain is one of the main symptoms of axSpA [5] and inflammatory back pain is a central criteria for disease diagnosis [3]. On the other hand, it is also well established that low back pain could significantly affect spatiotemporal gait parameters during walking [19-21]. For instance, previous studies have reported significant differences in spatiotemporal gait parameters between patients with low back pain and healthy matched controls $[19,20]$. Patients with low back pain presented lower gait speed [19,20] and cadence [20] and shorter stride length $[19,20]$ than healthy matched controls during walking. It is important to mention that self-reported pain intensity at time of evaluation was not reported in all studies on gait and AS. In particular, only studies from Mangone et al [17] and Zhang et al [17] have reported this parameter. Regardless of this, a careful examination and comparative analysis of these two published works $[17,18]$ nevertheless has drawn our attention to more specifically take into consideration the possible impact of pain on spatiotemporal gait parameters during walking. To support this view, let us first consider the work of Mangone et al [17]. Analysis of spatiotemporal gait showed no significant between-group difference for gait speed (AS: 0.94 [SD 0.2] $\mathrm{ms}^{-1}$ vs healthy controls: 0.96 [SD 0.2$\left.] \mathrm{ms}^{-1}, P=.78\right)$ and stride length (AS: 1.09 [SD 0.1] m vs healthy controls: 1.14 [SD 0.2] $\mathrm{m}, P=.40)$ [17]. Concomitantly, no between-group difference was observed for self-reported pain intensity reported at time of evaluation assessed with the visual analog scale (VAS-AS: 1.0 [SD 1.3] versus healthy controls: 0.7 [SD 1.1]) [17]. Worthy of note also are the very low self-reported pain intensity scores of close to 0 . A value of 0 on the VAS is considered as no pain while a value above 3 is considered as moderate pain [22]. In other words, participants of Mangone et al study [17] could thus be considered as pain-free participants.

Unlike the findings of Mangone et al [17], analysis of data from Zhang et al [18] revealed between-group significant difference in spatiotemporal gait. Lower gait speed (AS: 1.15 [SD 0.21] $\mathrm{ms}^{-1}$ vs healthy controls: 1.25 [SD 0.09] $\left.\mathrm{ms}^{-1}, P=.009\right)$ and shorter stride length (stride length/height: AS: 0.70 [SD 0.97] $\mathrm{m} / \mathrm{m}$ vs healthy controls: 0.76 [SD 0.42$] \mathrm{m} / \mathrm{m}, P=.002$ ) were observed in patients with AS $(n=18)$ than in healthy controls $(n=18)$ [18]. Meanwhile, pain intensity scores reported with the VAS in patients with AS only [18] were 3.89 [SD 1.64]. This value is above 3 and hence considered as moderate pain [22]. This self-reported pain intensity score is 3 times higher than that reported by patients with AS involved in the study of Mangone et al [17]. Although self-reported pain intensity was not collected in healthy controls, it is probable that the value for healthy controls would have been close to 0 like in Mangone 
et al [17] study. Taken together, the two studies have prompted us to consider that inconsistent and inconclusive results on gait in AS could stem from self-reported pain intensity at the time of the evaluation per se. Moreover, inertial measurement units (IMUs) are becoming helpful to assess gait in different populations $[23,24]$. IMUs allow computation of spatiotemporal gait parameters in clinical practice that are reliable in patients with axSpA. While previous studies demonstrated the advantages of using IMUs in axSpA to assess spinal mobility [25] or level of physical activity [26], no study assessed gait parameters using IMUs in patients with axSpA.

Overall, because of the lack of published works available on gait in patients with axSpA [13] and considering the divergent results obtained in the studies that have assessed spatiotemporal gait parameters in AS [14], which could be partly explained by self-reported pain intensity scores, this study was designed to compare spatiotemporal gait parameters in patients with axSpA and matched healthy individuals without and with pain intensity score as a covariate.

\section{Methods}

\section{Study Design}

The Function, Locomotion, Measurement, Inflammation (FOLOMI) study was approved by local ethics committee (CPP
Ile De France 1, RCB: 2017-A03468-45) and registered with ClinicalTrials.gov [NCT03761212] and followed the SPIRIT (Standard Protocol Items: Recommendations for Interventional Trials) checklist. All participants of the study signed informed consent.

\section{Participants}

The sample size of this study was calculated using difference between patients with AS and healthy controls in stride length in the Zebouni et al [16] study with a standard deviation of 0.12 , expected difference of 0.14 , significance level of 0.05 , and power of $80 \%$. The sample size was estimated at 12 in each group using a sample size calculator $[27,28]$. It was increased to 30 to allow the use of parametric tests.

Data for this cross-sectional study are a subset of individuals recruited in the FOLOMI prospective study that has been described in a previous publication [29]. The first 30 patients with axSpA included in FOLOMI study and 30 age- and sex-matched healthy controls were studied in this work. Inclusion and noninclusion criteria of the FOLOMI study are detailed below for patients with axSpA and for healthy controls in Textboxes 1 and 2.

Textbox 1. Patients with axSpA. axSpA: axial spondyloarthritis

Inclusion criteria:

- $\quad$ aged 18 to 65 years at time of their first evaluation

- $\quad \operatorname{axSpA}$ (based on ASAS criteria [3] or AS (based on modified New York Criteria [30])

- $\quad$ able to walk $180 \mathrm{~m}$ without technical help

- $\quad$ with stable treatment for 3 months

- $\quad$ with a public health insurance (French social security)

Exclusion criteria:

- musculoskeletal, cardiorespiratory or neurologic disease that could affect gait

- $\quad$ hip or knee arthroplasty done or planned in the following 18 months

- not able to speak French

- $\quad$ desire of pregnancy in the following 18 months

- $\quad$ adults protected by laws (Article L1121-5)

Textbox 2. Healthy controls.

Inclusion criteria:

- $\quad$ aged 18 to 65 years at time of evaluation

- $\quad$ able to walk $180 \mathrm{~m}$ without technical help

- $\quad$ with a health insurance

Exclusion criteria:

- musculoskeletal, cardiorespiratory or neurologic disease that could affect gait

- $\quad$ hip or knee arthroplasty done

- not able to speak French 


\section{Clinical Characteristics of the Participants}

Age, sex, weight, height, self-reported pain intensity at time of evaluation, and pain location were collected for both patients with axSpA and healthy controls by the same observer (JS) [29]. Self-reported pain intensity at time of evaluation score was assessed with the VAS, a horizontal line of $10 \mathrm{~cm}$ in length, anchored by word descriptors with no pain on the left side and the worst imaginable pain on the right side [22]. Participants were asked to mark the point corresponding to their current pain. Participants were asked to localized their pain using a pain areas figure [31].

For patients with axSpA only, disease clinical characteristics including treatment, disease duration, and morning stiffness and self-assessment questionnaires including the Bath Ankylosing Spondylitis Functional Index (BASFI) [32] and the Bath Ankylosing Spondylitis Disease Activity Index (BASDAI) [33] were also collected [29].

\section{Experimental Protocol}

Participants performed gait assessments described in a previous publication [29]. In this study, data from the 10-meter walk test (10MWT) in single-task condition only were considered. Participants performed a 10MWT on a 14-meter walkway at comfortable walking speed [34] in single-task condition (3 trials). Gait assessments were performed by the same examiner (JS). Participants wore walking shoes, with 2 inertial measurement units with triaxial accelerometers and gyroscopes (Physilog5, Gait Up), placed above both feet (behind the base of the fifth metatarsal) [35]. The two first and last steps were removed from the analysis [36,37], and at least 16 steps were included in the analysis. For patients with axSpA, regarding the possible consequences of morning stiffness on functional limitations [38], gait assessment was performed at least 2 hours from the end of morning stiffness.

\section{Spatiotemporal Gait Outcomes}

After checking for nonsignificant differences between left and right feet, the following spatiotemporal gait parameters were computed using Gait Analysis Software (version 5.3.0, Gait Up) with the mean of right and left foot values for each trial:

- $\quad$ Speed $\left(\mathrm{ms}^{-1}\right)$ : mean walking stride velocity of forward walking

- Cadence (steps/minute): number of steps in a minute

- Stride length $(\mathrm{m})$ : distance between two consecutive footprints on the ground, from the heel of a foot to the heel of the same foot, one cycle after

- Swing time (\%): portion of the cycle during which the foot is in the air and does not touch the ground
- Stance time (\%): portion of the cycle during which part of the foot touches the ground

- Double support time (\%): portion of the cycle where both feet touch the ground

The mean between trial 2 and 3 was calculated for each spatiotemporal gait parameter as it has recently been shown to be the more reliable to assess spatiotemporal gait parameters when performing a 10MWT at comfortable speed [35].

\section{Data Analysis}

Data analysis were performed using SPSS (version 20, IBM Corp) and Excel (Microsoft Corp). Independent samples $t$ tests were used to compare patients with axSpA and healthy controls in terms of age, gender, height, weight, self-reported pain intensity scores, and spatiotemporal gait parameters.

In the interest of further discerning differences that could exist as a function of group versus changes in self-reported pain intensity scores, the spatiotemporal gait parameters were further analyzed between groups using 1-way analyses of covariance (ANCOVAs) with the addition of pain intensity score as a covariate. Statistical threshold for all analyses was set at $P=.05$. Effect size (Cohen $d$ and partial $\eta^{2}$ ) and 95\% confidence intervals were also calculated.

\section{Results}

\section{Demographic and Clinical Assessments}

Demographic and clinical assessments for patients with axSpA and healthy controls are shown in Table 1. When comparing patients with axSpA and healthy controls, there were no significant differences for age, height, or weight, but patients with axSpA had higher self-reported pain intensity $(P<.001$; Table 1). In healthy controls, pain was located at the low back $(1 / 30,3 \%)$, knees $(1 / 30,3 \%)$, or shoulders $(1 / 30,3 \%)$. In patients with axSpA, pain was located at the low back (17/30, $57 \%)$, bottom or sacroiliac joints $(12 / 30,40 \%)$, thoracic back $(11 / 30,37 \%)$, cervical back $(14 / 30,47 \%)$, sternum or ribs $(1 / 30$, $3 \%)$, hips $(6 / 30,20 \%)$, knees $(9 / 30,30 \%)$, ankle or feet $(3 / 30$, $10 \%)$, shoulders $(6 / 30,20 \%)$, elbows $(4 / 30,13 \%)$, or hands $(5 / 30,17 \%)$.

Table 1 also presents pharmacological treatments and disease characteristics for patients with axSpA. Most patients with axSpA included in this study had anti-TNF treatment (21/30, 70\%), low disease activity with BASDAI <4 (BASDAI: 3.04 [SD 1.90]), and low impact of axSpA on physical function (BASFI: 2.86 [SD 2.04]). 
Table 1. Patients with axSpA and healthy controls clinical characteristics $(n=60)$.

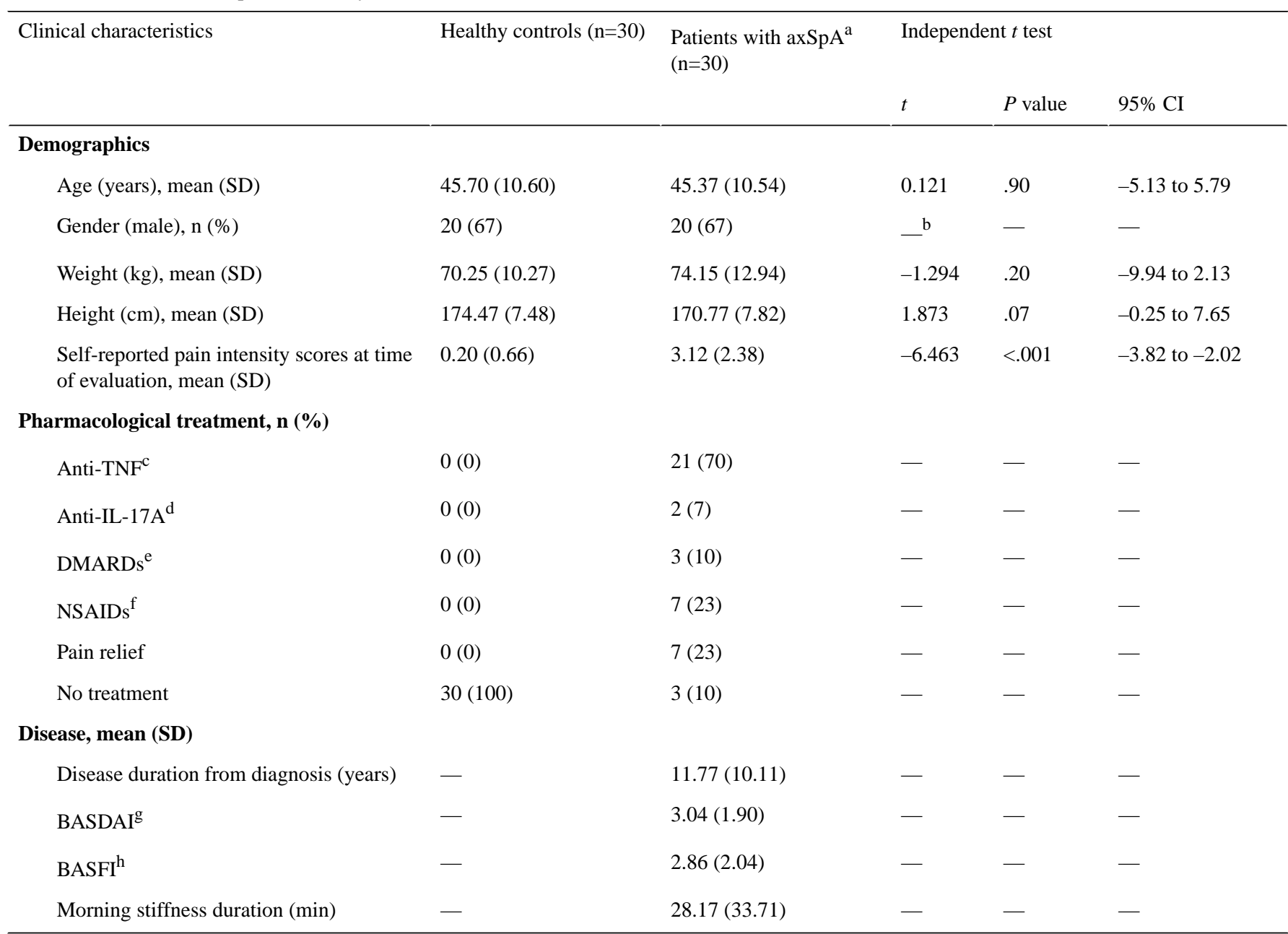

axSpA: axial spondyloarthritis.

${ }^{\mathrm{b}}$ Not applicable.

${ }^{\mathrm{c}}$ Anti-TNF: antitumor necrosis factor.

d Anti-IL-17A: anti-interleukine-17A.

e DMARD: disease modifying antirheumatic drug.

${ }^{f}$ NSAID: nonsteroidal anti-inflammatory agent.

g BASDAI: Bath Ankylosing Spondylitis Disease Activity Index.

${ }^{h}$ BASFI: Bath Ankylosing Spondylitis Functional Index.

\section{Spatiotemporal Gait Parameters}

Spatiotemporal gait parameters for patients with axSpA and healthy controls are shown in Table 2. Independent sample $t$ tests without covariate indicated that patients with axSpA presented lower gait speed $(P<.001)$ and cadence $(P=.004)$, shorter stride length $(P<.001)$ and swing time $(P<.001)$, and longer double support time $P<.001)$ and stance time $(P<.001)$ than matched healthy controls (Table 2).

ANCOVA comparisons of spatiotemporal gait parameters between groups revealed that a significant effect of group was found ( $F$ : 3.434, $P=.004$, partial $\eta^{2}$ : 0.320). Results for each spatiotemporal gait parameter can be found in Table 2. When using self-reported pain intensity score as a covariate, spatiotemporal gait parameters were still significant with patients with axSpA exhibiting lower gait speed $(P<.001)$, shorter stride length $(P=.001)$ and swing time $(P<.001)$, and longer double support time $(P<.001)$ and stance time $(P<.001)$ than matched healthy controls except for cadence which was not significant $(P=.17$; Table 2). 
Table 2. Spatiotemporal gait parameters obtained in patients with axSpA and healthy controls in single-task condition with $t$ test and ANCOVA results when taking self-reported pain intensity as a covariate.

\begin{tabular}{|c|c|c|c|c|c|c|c|c|c|}
\hline \multirow{2}{*}{$\begin{array}{l}\text { Spatiotemporal gait } \\
\text { parameters }\end{array}$} & \multirow{2}{*}{$\begin{array}{l}\text { Healthy controls } \\
(n=30) \text {, mean }(S D)\end{array}$} & \multirow{2}{*}{$\begin{array}{l}\text { Patients with axSpA } \\
(\mathrm{n}=30), \text { mean }(\mathrm{SD})\end{array}$} & \multicolumn{4}{|c|}{ Independent $t$ test } & \multicolumn{3}{|c|}{ ANCOVA $^{\mathrm{b}}$} \\
\hline & & & $t$ & $P$ value & Cohen $d$ & $95 \% \mathrm{CI}$ & $F$ & $P$ value & Partial $\eta^{2}$ \\
\hline Speed $\left(\mathrm{ms}^{-1}\right)$ & $1.50(0.16)$ & $1.27(0.17)$ & 5.528 & $<.001$ & 1.17 & 0.15 to 0.32 & 15.268 & $<.001$ & 0.211 \\
\hline Cadence (steps/min) & $113.89(6.35)$ & $108.41(7.85)$ & 2.97 & .004 & 0.72 & 1.79 to 9.17 & 1.922 & .17 & 0.033 \\
\hline Stride length (m) & $1.56(0.14)$ & $1.38(0.15)$ & 4.679 & $<.001$ & 1.04 & 0.10 to 0.25 & 13.508 & .001 & 0.192 \\
\hline $\begin{array}{l}\text { Double support time } \\
(\%)\end{array}$ & $19.43(3.42)$ & $22.99(2.50)$ & -4.609 & $<.001$ & -1.03 & -5.11 to -2.01 & 13.948 & $<.001$ & 0.197 \\
\hline Swing time (\%) & $39.84(1.77)$ & $38.20(1.19)$ & 4.201 & $<.001$ & 0.96 & 0.86 to 2.41 & 14.011 & $<.001$ & 0.197 \\
\hline Stance time $(\%)$ & $60.16(1.77)$ & $61.80(1.19)$ & -4.201 & $<.001$ & -0.96 & -2.41 to -0.86 & 14.011 & $<.001$ & 0.197 \\
\hline
\end{tabular}

a axSpA: axial spondyloarthritis.

b ANCOVA: one-way analysis of covariance using pain as covariate.

\section{Discussion}

\section{Principal Findings}

Only a few studies have assessed gait in the broader spectra of axSpA [13,26,39]. What is more, these studies have used clinical measurements of gait (ie, 6-min walk test [26] or 6-meter maximum velocity test [39]) without a healthy control group for comparison. Inconsistent results were found in patients with AS regarding spatiotemporal gait parameters [15-18], which may be explained by the rather small sample sizes of these studies and by self-reported pain intensity scores reported by the patients at the time of the evaluation.

This study was hence specifically designed to evaluate and compare spatiotemporal gait in 30 patients with axSpA and 30 matched healthy controls without and with pain intensity score as a covariate.

We found that patients with axSpA walked with reduced speed, cadence, stride length, and swing time and increased double support and stance time and that pain could per se partly explain this gait behavior. These results are in line with those recently reported by Zhang et al [18]. However, it should be noted that we further broaden the range of patients by including patients with axSpA, including AS and nr-axSpA, while Zhang et al [18] assessed gait in patients with AS and with hip involvement only. To our knowledge, this is the first study comparing spatiotemporal gait parameters in the broad range of patients with axSpA and matched healthy individuals [13]. Zhang et al [18] used a 3D motion-capture system, which is hardly accessible to clinical routine, while we used IMUs positioned on the feet, allowing computation of spatiotemporal gait parameters in clinical practice or in an ecological environment $[23,40]$. Finally, contrary to the Zhang et al [18] study, we included pain as a covariate to examine whether and to what extent self-reported pain intensity score could explain the gait differences observed between patients with axSpA and healthy controls.

Our results first showed a significant decrease of gait speed (control: 1.50 [SD 0.16] vs axSpA: 1.27 [SD 0.17] $\mathrm{m} / \mathrm{s}$, $\Delta=-16.6 \%, P<.001)$ of patients with axSpA as compared to matched healthy controls. This statistically significant difference is accompanied by a Cohen $d$ effect size of 1.17 , hence suggesting that the between groups difference for the gait speed is large $(d>0.8)$ [41]. In the absence of published work on gait in patients with axSpA [13] and although the included population was broader (axSpA vs AS), we were inclined to compare our results with those obtained in patients with AS. With this in mind, our result is in line with that reported in patients with AS by Zhang et al [18], who compared 18 patients with AS to 18 healthy matched controls (control: 1.25 [SD 0.09] vs AS: 1.15 [SD 0.21$] \mathrm{m} / \mathrm{s}, \Delta=-8.3 \%, P=.009)$. Gait speed of patients and healthy controls measured in this study was slightly higher than that reported in Zhang et al [18] (this study axSpA: 1.27 [SD 0.17] vs Zhang et al [18] AS: 1.15 [SD 0.21] $\mathrm{m} / \mathrm{s}$, $\Delta=-9.9 \%$; this study control: 1.50 [SD 0.16] vs Zhang et al [18] control: 1.25 [SD 0.09$] \mathrm{m} / \mathrm{s}, \Delta=-16.6 \%$ ). If gait were assessed along 10 meters in both studies, Zhang et al [18] included gait initiation, steady-state walking, and gait termination in the analysis. In our study, the acceleration and deceleration phases achieved during gait initiation and termination were not included. We used a 14-meter walkway [42-44] and removed the two first and the two last steps of the trials [36,37], as previously proposed in other studies that have assessed spatiotemporal gait parameters during walking [45-47]. When compared to other studies on AS, our result on gait speed does not corroborate those of Del Din et al [15] (12 AS vs 12 controls, control: 1.12 [SD 0.25] vs AS: 1.05 [SD 0.23$] \mathrm{m} / \mathrm{s}, \Delta=-6.45 \%$, $P=.33$ ) and Mangone et al [17] (17 AS vs 10 controls, control: 0.96 [SD 0.2] vs AS: 0.94 [SD 0.2] $\mathrm{m} / \mathrm{s}, \Delta=-2.1 \%, P=.78$ ), who did not report any significant between-group differences for the gait speed.

Our results further showed a significantly shorter stride length in patients with axSpA than in matched healthy controls (control: 1.56 [SD 0.14] vs axSpA: 1.38 [SD 0.15] $\mathrm{m}, \Delta=-12.2 \%, P<.001$ ) with a large Cohen $d$ effect size of 1.04. This result is in agreement with the decrease in stride length of patients with AS observed in two previous studies by Zebouni et al [16] (12 AS vs 11 controls, control: 0.72 [SD 0.13] vs AS: 0.58 [SD $0.11] \mathrm{m}, \Delta=-21.5 \%, P<.05$ ) and Zhang et al [18] (stride length/height: control: 0.76 [SD 0.42], AS: 0.70 [SD 0.97], 
$\Delta=-8.2 \%, P=.002)$. However, our result is not in line with two other studies on AS by Del Din et al [15] and Mangone et al [17], who did not report any significant differences in stride length between AS and controls (control: 1.29 [SD 0.30] vs AS: 0.98 [SD 0.58] m, $\Delta=-27.3 \%, P=.27$ [15]; control: 1.14 [SD $0.2]$ vs AS: 1.09 [SD 0.1] $\mathrm{m}, \Delta=-4.48 \%, P=.40$ [17]).

Our results further revealed a significant reduction of cadence in patients with axSpA as compared to matched healthy controls (control: 113.89 [SD 6.35] vs axSpA: 108.41 [SD 7.85] steps $/ \mathrm{min}, \Delta=-4.9 \%, P=.004$ ) with a medium effect size (Cohen $d: 0.72$ ). This result does not support the previous findings of Zhang et al [18], Zebouni et al [16], or Mangone et al [17], as no significant difference of cadence between patients with AS and healthy controls was observed (control: 0.94 [SD 0.04] vs AS: 0.95 [SD 0.09] /s, $\Delta=1.06 \%, P=.601$ [18]; control: 103.2 [SD 6.6] vs AS: 102.6 [SD 9] steps $/ \mathrm{min}, \Delta=-0.58 \%$, $P=$ nonsignificant [16]; control: 101.4 [SD 8.7] vs AS: 102.4 [SD 13.3] steps/min, $\Delta=0.98 \%, P=.65$ [17]).

In addition to these three routinely used spatiotemporal gait parameters, we further computed temporal distribution of gait cycle phases using swing time, stance time, and double support time percentages. The distribution of swing and stance period are temporal indicators of gait pattern [48] and often used as objectives in gait rehabilitation [49]. Indeed, the percentage times spent on swing and stance phases are determined by various factors including balance [50] and push-off force generation responsible for step asymmetry in chronic hemiparesis [51] and are associated with gait speed [52]. Only one study on patients with AS assessed these two temporal parameters [18]. Our results showed shorter swing time percentages (control: $39.84 \%$ [SD $1.77 \%$ ] vs axSpA: $38.20 \%$ [SD $1.19 \%$ ] of gait cycle, $\Delta=-4.2 \%, P<.001$, Cohen $d$ : 0.96) and longer stance time percentages (control: 60.16\% [SD 1.77\%] vs axSpA: $61.8 \%$ [SD $1.19 \%$ ] of gait cycle, $\Delta=2.69 \% ; P<.001$, Cohen $d$ : -0.96$)$ in patients with axSpa than matched healthy controls. Once again, our results are not in agreement with the existing literature as no significant difference with healthy controls of swing period was found by Zhang et al [18] (right: control: $38.61 \%$ [SD 1.55\%] vs AS: $38.29 \%$ [SD 2.62\%] of gait cycle, $\Delta=-0.83 \%, P=.64$; left: control: $38.49 \%$ [SD $1.66 \%$ ] vs AS: $38.12 \%$ [SD $3.95 \%$ ] of gait cycle, $\Delta=-0.97 \%, P=.57$ ).

Our results further showed longer double support time percentages in patients with axSpa than matched healthy controls (control: $19.43 \%$ [SD 3.42\%] vs axSpA: $22.99 \%$ [SD 2.5\%] of gait cycle, $\Delta=16.8 \%, P<.001$, Cohen $d:-1.03)$. Note that the Cohen $d$ effect size for double support time can be considered as large (>0.8). Interestingly, double support time percentage values obtained in this study cannot be compared to other studies as this parameter has never been assessed in AS [14].

To conclude, both the results of this study and those published elsewhere revealed a remarkable lack of consensus in the academic literature on gait and AS, although the low number of published studies and various methodologies make comparisons rather difficult. What explanation could we have for these differences?
Note that the demographic and clinical characteristics of patients and healthy controls (age, weight, and height) and disease duration of patients with axSpA involved in this study (age: 45 years, disease duration: 11.77 years) were comparable to those reported in previous studies (age between 38 and 49.4 years [15-18]; disease duration between 9.3 and 15 years [15-18]) and hence may not account for the observed divergent results.

We further assessed if divergent results previously reported on gait in AS [14] could be partly explained by self-reported pain intensity score at the time of the evaluation per se. The second statistical analysis presented in this study showed that when adjusting for self-reported pain intensity, patients with axSpA still presented lower gait speed, shorter stride length and swing time, and longer double support time. Interestingly, our results also revealed that there were no longer statistically significant between-group differences observed for the cadence. Taken together, these results suggest that differences between groups on cadence observed in this study could thus stem from self-reported pain intensity at the time of the evaluation per se and could explain why previous studies in AS did not find significant differences in cadence $[16,17]$ and reported low pain intensity in patients [17]. In a complementary way, results also suggest that differences between groups on the other spatiotemporal gait parameters observed in this study could not stem from self-reported pain intensity at the time of the evaluation per se. In other words, conclusions should be made with caution with respect to the influence of pain. Whether or not self-reported pain intensity per se could play a role in gait impairment observed in patients with axSpA still remains an open, unresolved question.

To synthesize the findings, patients with axSpA presented lower gait speed and cadence, shorter stride length and swing time, and longer double support time and stance time than matched healthy controls during walking. Taken together and looked into as a whole, these changes in spatiotemporal gait parameters could be interpreted as the adoption of a more conservative or less destabilizing gait in patients with axSpA (Figure 1). These results represent the characteristically so-called cautious gait pattern commonly observed in older persons [53] but also in individuals with gait disorders (eg, patients with cerebellar ataxia [54], with sensory ataxia [54], adults with obesity [55-57], and with low back pain [19-21]). This typical characteristic of cautious gait has already been observed in patients with AS $[15,16]$. However, these studies found that stride length was significantly shortened [16] or found only "a trend towards reduction" in gait speed or stride length [15] in patients with AS as compared to controls. Overall, it has been emphasized that individuals compensate for their balance disorders and/or gait by being more cautious during walking. Hence, adopting a more conservative gait pattern, characterized in particular by a slow gait speed, shortened stride/step length, reduced cadence, and an increased time spent in double limb support could be viewed as an adaptation to ensure or increase stability and maintain a safe gait $[53,58]$. 
Figure 1. Illustration of a healthy gait and a cautious gait pattern characterized by reduced gait speed and cadence, shortened stride length, and increased double support time.
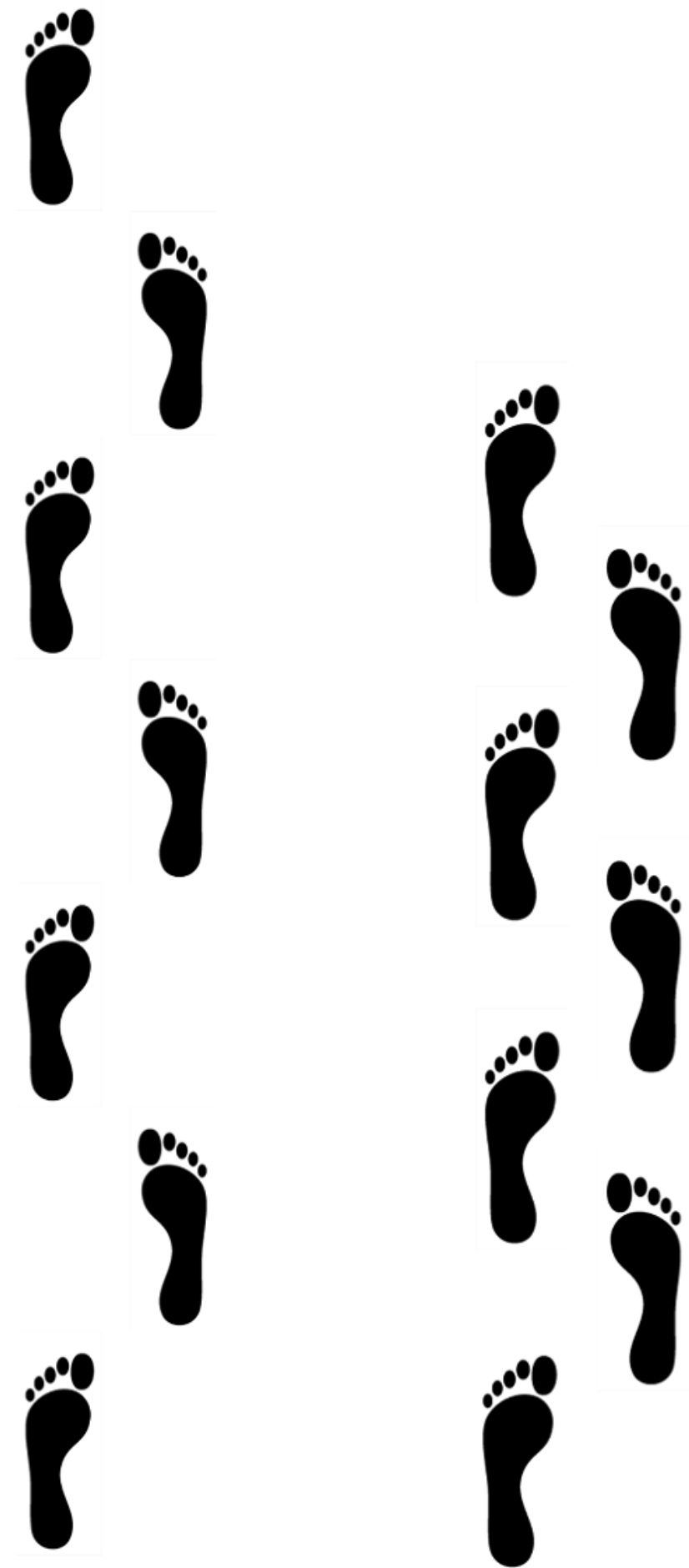

\section{HEALTHY GAIT}

It is important to note that the differences in spatiotemporal gait parameters obtained between patients with axSpA and healthy controls were outside the standard error of measurement and minimal detectable change (MDC), the minimum value for which a difference can be considered as real [59]. MDC adapted

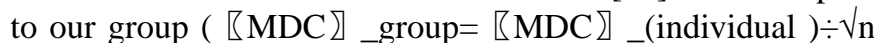
$[59,60])$ was 0.01 for speed and stride length, between 0.74 and 0.92 for cadence, between 0.24 and 0.53 for double support, and between 0.25 and 0.28 for swing time and stance time [35]. Accordingly, the significant between-group differences observed for these 6 spatiotemporal gait parameters cannot be considered as a measurement error. All in all, our findings showed that patients with axSpA adopted a cautious gait pattern in a similar fashion as the previously mentioned populations [20,53-57].

Taken together, the results of this study are promising for clinical application of gait analysis. We demonstrated that 
assessing gait in patients with AS using foot-worn inertial sensors is feasible in clinical settings. Spatiotemporal gait parameters (such as stride length or cadence) are the most used parameters in clinical gait analysis and are easy to understand by both clinicians [23] and patients. IMUs, by allowing rapid and easy-to-perform computation of spatiotemporal gait parameters at a low cost and without limitation of the testing environment, are gaining interests for clinicians [23,61]. The 10MWT used in this study is also routinely used by physiotherapists or medical doctors to evaluate gait in clinical and rehabilitation settings. In addition to the time taken to complete this test $[62,63]$, foot-worn inertial sensors enabled the quantitative gait patterns analysis of patients with axSpa with the computation of spatiotemporal gait parameters that were presented in an intuitive and comprehensible manner. We believe that integrating quantitative gait analysis with wearable IMU systems for clinical assessments could be advantageous for clinicians to better understand movement-related disorders for better functional diagnosis, guidance of treatment planning, monitoring of disease progress, and tracking of recovery [64]. In the near future, we can expect that mobile phone-based gait assessment apps will be used to monitor gait in daily life [65] and permit clinicians to remotely monitor patients' conditions $[66,67]$.

\section{Limitations}

Some limitations of the study should be acknowledged. First, patients included in the study were aged between 18 and 65 years with a pathology evolving with age and an increase of stiffness and limitations. Assessments of older patients could be interesting to capture gait alterations associated with disease evolution. Second, although self-reported pain intensity measured at time of assessment was significantly higher in patients with axSpA than healthy controls, levels of pain were quite low (3.12 [SD 2.38]). Patients included in this study were stable (ie, with stable treatment for at least 3 months at time of inclusion) and may not represent the whole population of patients with axSpA [68]. Further studies are thus necessary to explore gait in the broad disease of axSpA. Patient-reported pain intensity is commonly measured with the single VAS. However, VAS alone may not capture all features of pain $[69,70]$ and may be not sufficient to assess pain in patients with $\operatorname{axSpA~[71].~Finally,~additional~research~is~required~to~determine~}$ whether factors other than pain may influence gait in patients with axSpA.

\section{Conclusions}

To our knowledge, this is the first study comparing spatiotemporal gait parameters in a broad range of patients with axSpA and matched healthy individuals. Our results provide a comprehensive overview of the alterations of gait in patients with axSpA with reduced speed, cadence, stride length, and swing time and increased double support and stance. When all these changes in spatiotemporal gait parameters are taken together and looked into as a whole, it is possible to consider that patients with axSpA adopt a so-called cautious gait pattern. It is the first study to include pain intensity as a covariate to explain spatiotemporal gait parameters in patients with AS or axSpA. Although not a definitive finding, our results suggest that among factors that may influence gait in patients with axSpA, patient self-reported pain intensity could play a role and hence should be addressed when assessing gait in this population.

\section{Acknowledgments}

The authors would like to thank all the participants of the study and the FOLOMI (Function, Locomotion, Measurement, Inflammation) team for conceiving and designing the FOLOMI study (JS, NV, PG, AB, JV, Laurent Grange, Jean-Luc Cracowski, and Robert Juvin). We also would like to thank Thomas Carlin for his help on data analysis. This work is supported by grant PHRIP-16-0528 from the French Ministry of Health and Solidarity with the Nursing and Paramedical Hospital Research Program year 2016 and grants ANR-10-AIRT-05 and ANR-15-IDEX-02 from the French National Research Agency in the framework of the Investissements d'avenir program. The sponsors had no involvement in the design of the study; the collection, analysis, and interpretation of data; or in the writing of the manuscript. This study is part of the PhD thesis of the first author, JS (University Grenoble Alpes, Autonomy, Gerontology, E-health, Imaging and Stroke (AGEIS), France and Grenoble Alps University Hospital, Grenoble, France). This work further forms part of a broader translational and interdisciplinary research program GaitAlps (NV). Research data are available on request to the corresponding author.

\section{Authors' Contributions}

The FOLOMI (Function, Locomotion, Measurement, Inflammation) team conceptualized and designed the study [29]. JV and NV supervised the project. JS was responsible for the acquisition and analysis of the data. JS, JV, and NV interpreted the results. JS drafted the first version of the manuscript. JV and NV revised the article critically for important intellectual content. All authors read and approved the final version and agreed to be accountable for all aspects related to the accuracy or integrity of the work.

\section{Conflicts of Interest}

None declared.

\section{References}

1. Proft F, Poddubnyy D. Ankylosing spondylitis and axial spondyloarthritis: recent insights and impact of new classification criteria. Ther Adv Musculoskelet Dis 2018 Jun;10(5-6):129-139 [FREE Full text] [doi: 10.1177/1759720X18773726] [Medline: 29942364] 
2. Raychaudhuri SP, Deodhar A. The classification and diagnostic criteria of ankylosing spondylitis. J Autoimmun 2014 Feb;48-49:128-133. [doi: 10.1016/j.jaut.2014.01.015] [Medline: 24534717]

3. Rudwaleit M, van der Heijde D, Landewé R, Listing J, Akkoc N, Brandt J, et al. The development of Assessment of SpondyloArthritis international Society classification criteria for axial spondyloarthritis (part II): validation and final selection. Ann Rheum Dis 2009 Jun;68(6):777-783. [doi: 10.1136/ard.2009.108233] [Medline: 19297344]

4. Bohn R, Cooney M, Deodhar A, Curtis J, Golembesky A. Incidence and prevalence of axial spondyloarthritis: methodologic challenges and gaps in the literature. Clin Exp Rheumatol 2018;36(2):263-274 [FREE Full text] [Medline: 29148402]

5. Sieper J, Poddubnyy D. Axial spondyloarthritis. Lancet 2017 Jul 01;390(10089):73-84. [doi: 10.1016/S0140-6736(16)31591-4] [Medline: 28110981]

6. Bot S, Caspers M, Van Royen BJ, Toussaint H, Kingma I. Biomechanical analysis of posture in patients with spinal kyphosis due to ankylosing spondylitis: a pilot study. Rheumatology (Oxford) 1999 May;38(5):441-443. [doi:

10.1093/rheumatology/38.5.441] [Medline: 10371282]

7. Sato T, Yonezawa I, Inoue H, Tada K, Kobayashi S, Hayashi E, Juntendo AS Research Group. Relationship between characteristics of spinopelvic alignment and quality of life in Japanese patients with ankylosing spondylitis: a cross-sectional study. BMC Musculoskelet Disord 2020 Jan 18;21(1):41 [FREE Full text] [doi: 10.1186/s12891-020-3040-z] [Medline: 31954409]

8. Sahin N, Ozcan E, Baskent A, Karan A, Kasikcioglu E. Muscular kinetics and fatigue evaluation of knee using by isokinetic dynamometer in patients with ankylosing spondylitis. Acta Reumatol Port 2011;36(3):252-259 [FREE Full text] [Medline: 22113600]

9. Ro D, Lee J, Lee J, Park J, Han H, Lee M. Effects of knee osteoarthritis on hip and ankle gait mechanics. Adv Orthop 2019;2019:9757369 [FREE Full text] [doi: 10.1155/2019/9757369] [Medline: $\underline{\text { 31019809] }}$

10. Suh MJ, Kim BR, Kim SR, Han EY, Nam KW, Lee SY, et al. Bilateral quadriceps muscle strength and pain correlate with gait speed and gait endurance early after unilateral total knee arthroplasty: a cross-sectional study. Am J Phys Med Rehabil 2019 Oct;98(10):897-905. [doi: 10.1097/PHM.0000000000001222] [Medline: $\underline{\text { 31094710] }}$

11. Tanishima S, Hagino H, Matsumoto H, Tanimura C, Nagashima H. Association between sarcopenia and low back pain in local residents prospective cohort study from the GAINA study. BMC Musculoskelet Disord 2017 Nov 15;18(1):452 [FREE Full text] [doi: 10.1186/s12891-017-1807-7] [Medline: 29141602]

12. Otayek J, Bizdikian AJ, Yared F, Saad E, Bakouny Z, Massaad A, et al. Influence of spino-pelvic and postural alignment parameters on gait kinematics. Gait Posture 2020 Feb;76:318-326. [doi: 10.1016/j.gaitpost.2019.12.029] [Medline: 31891899]

13. Soulard J, Vaillant J, Vuillerme N. Gait in patients with axial spondyloarthritis: a systematic review of the literature. Curr Rheumatol Rev 2021 Sep 20:1. [doi: 10.2174/1573397117666210921114949] [Medline: 34548003]

14. Soulard J, Vaillant J, Agier C, Vuillerme N. Gait characteristics in patients with ankylosing spondylitis: a systematic review. Clin Exp Rheumatol 2021;39(1):173-186 [FREE Full text] [Medline: 33025884]

15. Del DS, Carraro E, Sawacha Z, Guiotto A, Bonaldo L, Masiero S, et al. Impaired gait in ankylosing spondylitis. Med Biol Eng Comput 2011 Jul;49(7):801-809. [doi: 10.1007/s11517-010-0731-x] [Medline: 21229328]

16. Zebouni L, Helliwell PS, Howe A, Wright V. Gait analysis in ankylosing spondylitis. Ann Rheum Dis 1992 Jul 01;51(7):898-899 [FREE Full text] [doi: 10.1136/ard.51.7.898] [Medline: 1632666]

17. Mangone M, Scettri P, Paoloni M, Procaccianti R, Spadaro A, Santilli V. Pelvis-shoulder coordination during level walking in patients with ankylosing spondylitis. Gait Posture 2011 May;34(1):1-5. [doi: 10.1016/j.gaitpost.2011.02.002] [Medline: 21377366]

18. Zhang G, Li J, Xia Z, Xu W. The gait deviations of ankylosing spondylitis with hip involvement. Clin Rheumatol 2019 Apr 4;38(4):1163-1175. [doi: 10.1007/s10067-018-4401-y] [Medline: 30607653]

19. Demirel A, Onan D, Oz M, Ozel Asliyuce Y, Ulger O. Moderate disability has negative effect on spatiotemporal parameters in patients with chronic low back pain. Gait Posture 2020 Jun;79:251-255. [doi: 10.1016/j.gaitpost.2020.05.015] [Medline: $\underline{32460134]}$

20. Bonab M, Colak TK, Toktas ZO, Konya D. Assessment of spatiotemporal gait parameters in patients with lumbar disc herniation and patients with chronic mechanical low back pain. Turk Neurosurg 2020;30(2):277-284 [FREE Full text] [doi: 10.5137/1019-5149.JTN.27499-19.2] [Medline: 32091127]

21. Hicks GE, Sions JM, Coyle PC, Pohlig RT. Altered spatiotemporal characteristics of gait in older adults with chronic low back pain. Gait Posture 2017 Jun;55:172-176 [FREE Full text] [doi: 10.1016/j.gaitpost.2017.04.027] [Medline: 28458149]

22. Williamson A, Hoggart B. Pain: a review of three commonly used pain rating scales. J Clin Nurs 2005 Aug; 14(7):798-804 [FREE Full text] [doi: 10.1111/j.1365-2702.2005.01121.x] [Medline: 16000093]

23. Iosa M, Picerno P, Paolucci S, Morone G. Wearable inertial sensors for human movement analysis. Expert Rev Med Devices 2016 Jul;13(7):641-659. [doi: 10.1080/17434440.2016.1198694] [Medline: 27309490]

24. Chen S, Lach J, Lo B, Yang G. Toward pervasive gait analysis with wearable sensors: a systematic review. IEEE J Biomed Health Inform 2016 Nov;20(6):1521-1537. [doi: 10.1109/JBHI.2016.2608720] [Medline: 28113185]

25. Aranda-Valera I, Cuesta-Vargas A, Garrido-Castro J, Gardiner P, López-Medina C, Machado P, et al. Measuring spinal mobility using an inertial measurement unit system: a validation study in axial spondyloarthritis. Diagnostics (Basel) 2020 Jun 24;10(6):426 [FREE Full text] [doi: 10.3390/diagnostics10060426] [Medline: $\underline{\text { 32599741] }}$ 
26. Coulter EH, McDonald MT, Cameron S, Siebert S, Paul L. Physical activity and sedentary behaviour and their associations with clinical measures in axial spondyloarthritis. Rheumatol Int 2020 Mar;40(3):375-381 [FREE Full text] [doi: 10.1007/s00296-019-04494-3] [Medline: $\underline{\text { 31848736] }}$

27. Lwanga S, Lemeshow S. Sample size determination in health studies : a practical manual. Geneva: World Health Organization; 1991. URL: https://apps.who.int/iris/handle/10665/40062 [accessed 2021-09-18]

28. Sample size calculator. URL: https://wnarifin.github.io/ssc/ss2mean.html [accessed 2021-09-18]

29. Soulard J, Vuillerme N, Gaudin P, Grange L, Baillet A, Cracowski J, et al. Gait as predictor of physical function in axial spondyloarthritis: the prospective longitudinal FOLOMI (Function, Locomotion, Measurement, Inflammation) study protocol. Rheumatol Int 2019 Oct;39(10):1681-1688. [doi: 10.1007/s00296-019-04396-4] [Medline: $\underline{31392500]}$

30. van der Linden S, Valkenburg HA, Cats A. Evaluation of diagnostic criteria for ankylosing spondylitis. A proposal for modification of the New York criteria. Arthritis Rheum 1984 Apr;27(4):361-368. [doi: 10.1002/art.1780270401] [Medline: $\underline{6231933}]$

31. Escalante A, Lichtenstein MJ, White K, Rios N, Hazuda HP. A method for scoring the pain map of the McGill Pain Questionnaire for use in epidemiologic studies. Aging (Milano) 1995 Oct;7(5):358-366. [doi: 10.1007/BF03324346] [Medline: 8719602$]$

32. Calin A, Garrett S, Whitelock H, Kennedy L, O'Hea J, Mallorie P, et al. A new approach to defining functional ability in ankylosing spondylitis: the development of the Bath Ankylosing Spondylitis Functional Index. J Rheumatol 1994 Dec;21(12):2281-2285. [Medline: 7699629$]$

33. El Miedany Y, Youssef S, Mehanna A, Shebrya N, Abu Gamra S, El Gaafary M. Defining disease status in ankylosing spondylitis: validation and cross-cultural adaptation of the Arabic Bath Ankylosing Spondylitis Functional Index (BASFI), the Bath Ankylosing Spondylitis Disease Activity Index (BASDAI), and the Bath Ankylosing Spondylitis Global score (BASG). Clin Rheumatol 2008 May 5;27(5):605-612. [doi: 10.1007/s10067-007-0755-2] [Medline: 18180979]

34. Graham JE, Ostir GV, Fisher SR, Ottenbacher KJ. Assessing walking speed in clinical research: a systematic review. J Eval Clin Pract 2008 Aug;14(4):552-562 [FREE Full text] [doi: 10.1111/j.1365-2753.2007.00917.x] [Medline: 18462283]

35. Soulard J, Vaillant J, Balaguier R, Baillet A, Gaudin P, Vuillerme N. Foot-worn inertial sensors are reliable to assess spatiotemporal gait parameters in axial spondyloarthritis under single and dual task walking in axial spondyloarthritis. Sensors (Basel) 2020 Nov 12;20(22):6453 [FREE Full text] [doi: 10.3390/s20226453] [Medline: $\underline{33198119]}$

36. Truong P, Lee J, Kwon A, Jeong G. Stride counting in human walking and walking distance estimation using insole sensors. Sensors (Basel) 2016 Jun 04;16(6):823 [FREE Full text] [doi: 10.3390/s16060823] [Medline: 27271634]

37. Anwary A, Yu H, Vassallo M. An automatic gait feature extraction method for identifying gait asymmetry using wearable sensors. Sensors (Basel) 2018 Feb 24;18(2):676 [FREE Full text] [doi: 10.3390/s18020676] [Medline: 29495299]

38. Song Y, Wang C, Chen H. Functional limitation and associated factors in outpatients with ankylosing spondylitis in Southwest China. Clin Rheumatol 2017 Apr;36(4):871-877. [doi: 10.1007/s10067-017-3563-3] [Medline: 28168660]

39. Eppeland S, Diamantopoulos AP, Soldal D, Haugeberg G. Short term in-patient rehabilitation in axial spondyloarthritis: the results of a 2-week program performed in daily clinical practice. BMC Res Notes 2013 May 07;6(1):185 [FREE Full text] [doi: 10.1186/1756-0500-6-185] [Medline: 23651526]

40. Kavanagh JJ, Menz HB. Accelerometry: a technique for quantifying movement patterns during walking. Gait Posture 2008 Jul;28(1):1-15. [doi: 10.1016/j.gaitpost.2007.10.010] [Medline: 18178436 ]

41. Cohen J. Statistical Power Analysis for the Behavioral Sciences. 2nd Edition, reprint. New York: Psychology Press; 2009.

42. Cheng DK, Nelson M, Brooks D, Salbach NM. Validation of stroke-specific protocols for the 10-meter walk test and 6-minute walk test conducted using 15-meter and 30-meter walkways. Top Stroke Rehabil 2020 May;27(4):251-261. [doi: 10.1080/10749357.2019.1691815] [Medline: 31752634]

43. Chan WL, Pin TW. Reliability, validity and minimal detectable change of 2-minute walk test, 6-minute walk test and 10-meter walk test in frail older adults with dementia. Exp Gerontol 2019 Jan;115:9-18. [doi: 10.1016/j.exger.2018.11.001] [Medline: 30423359 ]

44. Nascimento LR, Caetano LCG, Freitas DCMA, Morais TM, Polese JC, Teixeira-Salmela LF. Different instructions during the ten-meter walking test determined significant increases in maximum gait speed in individuals with chronic hemiparesis. Rev Bras Fisioter 2012 Apr;16(2):122-127. [doi: 10.1590/s1413-35552012005000008] [Medline: 22378478]

45. Graser JV, Letsch C, van Hedel HJA. Reliability of timed walking tests and temporo-spatial gait parameters in youths with neurological gait disorders. BMC Neurol 2016 Jan 31;16(1):15 [FREE Full text] [doi: 10.1186/s12883-016-0538-y] [Medline: 26830919]

46. Sorsdahl AB, Moe-Nilssen R, Strand LI. Test-retest reliability of spatial and temporal gait parameters in children with cerebral palsy as measured by an electronic walkway. Gait Posture 2008 Jan;27(1):43-50. [doi: 10.1016/j.gaitpost.2007.01.001] [Medline: 17300940]

47. Pérez-Sanpablo AI, Quinzaños-Fresnedo J, Loera-Cruz R, Quiñones-Uriostegui I, Rodriguez-Reyes G, Pérez-Zavala R. Validation of the instrumented evaluation of spatio-temporal gait parameters in patients with motor incomplete spinal cord injury. Spinal Cord 2017 Feb 28;55(7):699-704. [doi: 10.1038/sc.2017.4]

48. Mariani B, Rouhani H, Crevoisier X, Aminian K. Quantitative estimation of foot-flat and stance phase of gait using foot-worn inertial sensors. Gait Posture 2013 Feb;37(2):229-234. [doi: 10.1016/j.gaitpost.2012.07.012] [Medline: 22877845] 
49. Jayakaran P, DeSouza L, Cossar J, Gilhooly K. Influence of a walking aid on temporal and spatial parameters of gait in healthy adults. PM R 2014 Sep;6(9):796-801. [doi: 10.1016/j.pmrj.2014.02.005] [Medline: 24534098]

50. Lopes PG, Lopes JAF, Brito CM, Alfieri FM, Rizzo Battistella L. Relationships of balance, gait performance, and functional outcome in chronic stroke patients: a comparison of left and right lesions. Biomed Res Int 2015;2015:716042-716048 [FREE Full text] [doi: 10.1155/2015/716042] [Medline: 26583129]

51. Balasubramanian CK, Bowden MG, Neptune RR, Kautz SA. Relationship between step length asymmetry and walking performance in subjects with chronic hemiparesis. Arch Phys Med Rehabil 2007 Jan;88(1):43-49. [doi: 10.1016/j.apmr.2006.10.004] [Medline: 17207674]

52. Wang Y, Mukaino M, Ohtsuka K, Otaka Y, Tanikawa H, Matsuda F, et al. Gait characteristics of post-stroke hemiparetic patients with different walking speeds. Int J Rehabil Res 2020 Mar;43(1):69-75 [FREE Full text] [doi: 10.1097/MRR.0000000000000391] [Medline: 31855899]

53. Herssens N, Verbecque E, Hallemans A, Vereeck L, Van Rompaey V, Saeys W. Do spatiotemporal parameters and gait variability differ across the lifespan of healthy adults? A systematic review. Gait Posture 2018 Jul;64:181-190. [doi: 10.1016/j.gaitpost.2018.06.012] [Medline: 29929161]

54. Pirker W, Katzenschlager R. Gait disorders in adults and the elderly: a clinical guide. Wien Klin Wochenschr 2017 Feb;129(3-4):81-95 [FREE Full text] [doi: 10.1007/s00508-016-1096-4] [Medline: 27770207]

55. Lim J, Kim J, Seo K, van Emmerik REA, Lee S. The effects of mobile texting and walking speed on gait characteristics of normal weight and obese adults. Motor Control 2020 Sep 10:1-17. [doi: 10.1123/mc.2020-0006] [Medline: 32916659]

56. Maktouf W, Durand S, Boyas S, Pouliquen C, Beaune B. Interactions among obesity and age-related effects on the gait pattern and muscle activity across the ankle joint. Exp Gerontol 2020 Oct 15;140:111054. [doi: 10.1016/j.exger.2020.111054] [Medline: $\underline{\text { 32791335] }}$

57. McGraw B, McClenaghan BA, Williams HG, Dickerson J, Ward DS. Gait and postural stability in obese and nonobese prepubertal boys. Arch Phys Med Rehabil 2000 Apr;81(4):484-489. [doi: 10.1053/mr.2000.3782] [Medline: 10768540]

58. Cromwell RL, Newton RA. Relationship between balance and gait stability in healthy older adults. J Aging Phys Act 2004 Jan;12(1):90-100. [doi: 10.1123/japa.12.1.90] [Medline: 15211023]

59. Washabaugh EP, Kalyanaraman T, Adamczyk PG, Claflin ES, Krishnan C. Validity and repeatability of inertial measurement units for measuring gait parameters. Gait Posture 2017 Jun;55:87-93 [FREE Full text] [doi: 10.1016/j.gaitpost.2017.04.013] [Medline: 28433867]

60. Lu W, Wang C, Lin J, Sheu C, Hsieh C. The minimal detectable change of the simplified stroke rehabilitation assessment of movement measure. J Rehabil Med 2008 Aug;40(8):615-619 [FREE Full text] [doi: 10.2340/16501977-0230] [Medline: 19020694]

61. Muro-de-la-Herran A, Garcia-Zapirain B, Mendez-Zorrilla A. Gait analysis methods: an overview of wearable and non-wearable systems, highlighting clinical applications. Sensors (Basel) 2014 Feb 19;14(2):3362-3394 [FREE Full text] [doi: 10.3390/s140203362] [Medline: 24556672]

62. Amatachaya S, Kwanmongkolthong M, Thongjumroon A, Boonpew N, Amatachaya P, Saensook W, et al. Influence of timing protocols and distance covered on the outcomes of the 10-meter walk test. Physiother Theory Pract 2020 Dec;36(12):1348-1353. [doi: 10.1080/09593985.2019.1570577] [Medline: 30704332]

63. Lindholm B, Nilsson MH, Hansson O, Hagell P. The clinical significance of 10-m walk test standardizations in Parkinson's disease. J Neurol 2018 Aug;265(8):1829-1835 [FREE Full text] [doi: 10.1007/s00415-018-8921-9] [Medline: 29876762]

64. Baker R, Esquenazi A, Benedetti MG, Desloovere K. Gait analysis: clinical facts. Eur J Phys Rehabil Med 2016 Aug;52(4):560-574 [FREE Full text] [Medline: 27618499]

65. Lee S, Walker RM, Kim Y, Lee H. Measurement of human walking movements by using a mobile health app: motion sensor data analysis. JMIR Mhealth Uhealth 2021 Mar 05;9(3):e24194 [FREE Full text] [doi: 10.2196/24194] [Medline: 33666557]

66. Pratap A, Grant D, Vegesna A, Tummalacherla M, Cohan S, Deshpande C, et al. Evaluating the utility of smartphone-based sensor assessments in persons with multiple sclerosis in the real-world using an app (elevateMS): observational, prospective pilot digital health study. JMIR Mhealth Uhealth 2020 Oct 27;8(10):e22108 [FREE Full text] [doi: 10.2196/22108] [Medline: $\underline{33107827]}$

67. Muntaner-Mas A, Martinez-Nicolas A, Quesada A, Cadenas-Sanchez C, Ortega FB. Smartphone app (2kmFIT-App) for measuring cardiorespiratory fitness: validity and reliability study. JMIR Mhealth Uhealth 2021 Jan 08;9(1):e14864 [FREE Full text] [doi: 10.2196/14864] [Medline: $\underline{33416503}$ ]

68. Madsen OR. Stability of fatigue, pain, patient global assessment and the Bath Ankylosing Spondylitis Functional Index (BASFI) in spondyloarthropathy patients with stable disease according to the Bath Ankylosing Spondylitis Disease Activity Index (BASDAI). Rheumatol Int 2018 Mar 3;38(3):425-432. [doi: 10.1007/s00296-017-3920-1] [Medline: 29299630]

69. Kaciroti N, DosSantos MF, Moura B, Bellile EL, Nascimento TD, Maslowski E, et al. Sensory-discriminative three-dimensional body pain mobile app measures versus traditional pain measurement with a visual analog scale: validation study. JMIR Mhealth Uhealth 2020 Aug 19;8(8):e17754 [FREE Full text] [doi: 10.2196/17754] [Medline: 32124732]

70. Pathak A, Sharma S, Jensen MP. The utility and validity of pain intensity rating scales for use in developing countries. Pain Rep 2018;3(5):e672 [FREE Full text] [doi: 10.1097/PR9.0000000000000672] [Medline: 30534623] 
71. Duruöz MT, Acer Kasman S, Şahin N, Sezer, Bodur H, Ketenci A, Baklacıŏlu. Validity, reliability, and factor structure of the Istanbul Low Back Pain Disability Index in axial spondyloarthritis. Mod Rheumatol 2020 Mar 03:1-7. [doi: 10.1080/14397595.2020.1733172] [Medline: $\underline{\text { 32075459] }}$

\author{
Abbreviations \\ 10MWT: 10 -meter walk test \\ AGEIS: Autonomy, Gerontology, eHealth, Imaging, and Stroke \\ ANCOVA: one-way analysis of covariance \\ AS: ankylosing spondylitis \\ ASAS: Assessment of Spondyloarthritis International Society \\ axSpA: axial spondyloarthritis \\ BASDAI: Bath Ankylosing Spondylitis Disease Activity Index \\ BASFI: Bath Ankylosing Spondylitis Functional Index \\ FOLOMI study: Function, Locomotion, Measurement, Inflammation study \\ IMU: inertial measurement unit \\ nr-axSpA: nonradiographic axial spondyloarthritis \\ SpA: spondyloarthritis \\ SPIRIT: Standard Protocol Items: Recommendations for Interventional Trials \\ VAS: visual analog scale
}

\author{
Edited by L Buis; submitted 11.01.21; peer-reviewed by V Shah, Y Man; comments to author 10.05.21; revised version received \\ 18.05.21; accepted 23.07.21; published 09.11.21 \\ Please cite as: \\ Soulard J, Vaillant J, Baillet A, Gaudin P, Vuillerme N \\ Gait and Axial Spondyloarthritis: Comparative Gait Analysis Study Using Foot-Worn Inertial Sensors \\ JMIR Mhealth Uhealth 2021;9(11):e27087 \\ URL: https://mhealth.jmir.org/2021/11/e27087 \\ doi: $\underline{10.2196 / 27087}$ \\ PMID:
}

CJulie Soulard, Jacques Vaillant, Athan Baillet, Philippe Gaudin, Nicolas Vuillerme. Originally published in JMIR mHealth and uHealth (https://mhealth.jmir.org), 09.11.2021. This is an open-access article distributed under the terms of the Creative Commons Attribution License (https://creativecommons.org/licenses/by/4.0/), which permits unrestricted use, distribution, and reproduction in any medium, provided the original work, first published in JMIR mHealth and uHealth, is properly cited. The complete bibliographic information, a link to the original publication on https://mhealth.jmir.org/, as well as this copyright and license information must be included. 